\title{
Drug consumption in medication overuse headache is influenced by brain-derived neurotrophic factor Val66Met polymorphism
}

\author{
Cherubino Di Lorenzo · Giorgio Di Lorenzo - Grazia Sances - Natascia Ghiotto • \\ Elena Guaschino - Gaetano S. Grieco - Filippo M. Santorelli - Carlo Casali • \\ Alfonso Troisi · Alberto Siracusano $\cdot$ Francesco Pierelli
}

Received: 11 February 2009/Accepted: 20 May 2009/Published online: 11 June 2009

(C) Springer-Verlag 2009

\begin{abstract}
Medication overuse headache $(\mathrm{MOH})$ can be considered a clinical condition at the boundaries between drug addiction and chronic pain disorder. The common 196G > A single-nucleotide polymorphism of BDNF gene, resulting in a valine 66 to methionine (Val66Met), is related with behaviour disorders and substance abuse. With the aim of identifying a worsening factor in $\mathrm{MOH}$, rather
\end{abstract}

C. Di Lorenzo · F. Pierelli

University Center for Adaptive Disorders and Headache

(UCADH), University of Rome "La Sapienza",

Polo Pontino, ICOT, Latina, Italy

G. Di Lorenzo - A. Troisi - A. Siracusano

Psychiatric Clinic, Department of Neuroscience,

University of Rome "Tor Vergata”, Rome, Italy

G. Sances - N. Ghiotto - E. Guaschino

Headache Unit, University Center for Adaptive Disorders and Headache (UCADH), IRCCS "C. Mondino Institute

of Neurology" Foundation, Pavia, Italy

\section{G. S. Grieco}

Molecular Genetics Laboratory, IRCCS “C. Mondino Institute of Neurology" Foundation, Pavia, Italy

F. M. Santorelli

Molecular Medicine, IRCCS “Bambino Gesù”, Rome, Italy

C. Casali

Rehabilitation Unit, University of Rome "La Sapienza",

Polo Pontino, Latina, Italy

F. Pierelli

Headache Clinic, IRCCS INM Neuromed, Pozzilli (IS), Italy

C. Di Lorenzo $(\square)$

via Franco Faggiana, 34, 04100 Latina, Italy

e-mail: cherub@inwind.it than the detection of a specific risk factor for the development of the disease, we investigated whether the presence of a functional BDNF polymorphism might determine clinical differences within a group of $90 \mathrm{MOH}$ patients, particularly in monthly drug consumption, that is the hallmark of disease. Directly comparing $\mathrm{MOH}$ patients homozygous for $\mathrm{G}$ allele $(\mathrm{G} / \mathrm{G})$ with carriers of A allele (non-G/G), we have observed $47 \mathrm{G} / \mathrm{G}$ genotypes and 60 non-G/G genotypes. Non-G/G had a higher consumption of monthly drug number (Cohen's $d=0.76$ ) than $\mathrm{G} / \mathrm{G}$ patients. At multiple regression analysis, the Val66Met BDNF polymorphism emerged as a significant independent predictor of analgesic drug consumption (Beta $=0.33$, Cohen's $\left.f^{2}=0.134\right)$. These findings showed an influence of examined BDNF polymorphism in the $\mathrm{MOH}$ clinical features, supporting the idea that $\mathrm{MOH}$ is a substance abuse disorder.

Keywords Brain-derived neurotrophic factor (BDNF) . Genetic polymorphisms - Medication overuse headache $(\mathrm{MOH}) \cdot$ Addictive behaviour

\section{Introduction}

Medication overuse headache (MOH) is a chronic headache disorder due to overuse of analgesic medicines, taken to treat a primary headache, and remitted by drug discontinuation [1]. MOH has long been considered as a "biobehavioural" disorder, in which the biological predisposition to develop a chronic headache interacts with an individual behaviour prone to develop a dependence disorder [2,3]. The molecular basis of $\mathrm{MOH}$ are largely unknown, though it has been hypothesized that this subtype of headache could be mediated by cognitive impulsivity, and shares some 
pathophysiological mechanisms with drug addiction, including dysfunction of the frontostriatal system and central pain sensitization induced by drug overuse [4]. Based on this hypothesis, there seems to be a role in $\mathrm{MOH}$ for BDNF (brain-derived neurotrophic factor), because of its role in central pain sensitization and its relationship with substance-related disorders [5, 6].

BDNF is a member of "neurotrophin" family, a unique group of polypeptide growth factors that influence differentiation and survival of neurons in the developing nervous system. BDNF is involved in long-term potentiation (LTP), and therefore in learning and memory mechanisms. Also, a pathogenic role for BDNF has been shown in pain transduction and sensitization, in epilepsy, and in neuropsychiatric disorders [5].

BDNF gene, mapped to chromosome $11 \mathrm{p}$, encodes for pro-BDNF that is subsequently cleaved in its mature form [5]. Recently, it has been observed that a common 196G > A single-nucleotide polymorphism (SNP) of BDNF gene, resulting in a valine at residue 66 to methionine (Val66Met) substitution in pro-BDNF protein, exhibits a functional relevance in that it seems to reduce BDNF activity by altering neurotrophin trafficking [7]. The Val66Met variant affects anatomy, cognition and behaviour in adults [7] and leads to memory impairment [5] and susceptibility to obsessive-compulsive, eating, substance abuse and mood disorders [6, 8-10].

The rationale behind our study derives from two findings, which have been consistently reported in previous researches: (1) overuse of analgesic drugs is a defining feature of $\mathrm{MOH}$ and, for this reason, $\mathrm{MOH}$ has been related to behavioural syndromes associated with substance abuse [1-3], (2) there is evidence that proneness to use/abuse substances is associated with the allele A of BDNF polymorphism [6].Therefore, we tested the frequency of the Val66Met genotype in a sample of $\mathrm{MOH}$ patients, and compared clinical features between individuals homozygous for $\mathrm{G}$ allele $(\mathrm{G} / \mathrm{G})$ and carriers of the allele A (non-G/G). Aim of this study is to confirm our hypothesis that allele $\mathrm{A}$ of the examined BDNF polymorphism interfere with $\mathrm{MOH}$, seeking for clinical and psychiatric differences between the two groups, mainly in the monthly drug consumption, that is both the clinical hallmark of disease, and the feature closest to abuse disorder.

We would like to emphasize that, although such studies are necessary to get a deeper understanding of the association between $\mathrm{MOH}$ and BDNF polymorphism, the present study did not intend to ascertain whether the BDNF polymorphism is a risk factor for the development of $\mathrm{MOH}$, but only an aggravating factor for subjects that have already developed the disorder.

\section{Materials and methods}

Subjects and clinical data

Unrelated consecutive $\mathrm{MOH}$ patients were enrolled from two Italian University Headache Clinics: the inpatient Headache-Unit of IRCCS "C. Mondino" Institute of Neurology Foundation (Pavia), and Headache Outpatient Department of University of Rome "La Sapienza", among those admitted with a diagnosis of probable $\mathrm{MOH}$ [1], to undergo withdrawal from their overused medications. Although revised criteria were formulated for $\mathrm{MOH}$ [11], patients' enrolment was started before their statement, thus original ICHD-II version (i.e. in the presence of headache improvement within 2 months after analgesics withdrawal) [1], that is more conservative, was adopted to recruit all patients; a retrospective analysis will also confirm that all enrolled patients fulfilled the revised criteria.

Socio-demographic and clinical data were obtained by anamnesis; consumption of analgesic drugs per month (from here, monthly drug number), days with headache per month (from here, monthly headache days) and days with drug consumption per month (from here, monthly drug days) were self reported by patients through a headache diary, received at the time of first visit. Inclusion criteria were: (a) patient written informed consent, (b) absence of other major medical conditions, (c) absence of psychiatric disorders that could sustain a chronic pain condition (psychotic and/or somatoform disorders), (d) to have accurately completed the last 3-month pre-withdrawal diary, (e) ability to complete the psycho-diagnostic assessment, (f) diagnostic confirmation of MOH after withdrawal. Our Institutional Ethical Committees approved the study, conducted according to Declaration of Helsinki.

Trained interviewers, blinded to headache diagnosis, performed psychiatric assessment. Presence of categorical psychiatric diagnosis was investigated through four modules of the Structured Clinical Interview for DSM-IV Axis I Disorders (SCID-I), Clinician Version [12]: mood episodes, mood disorders, anxiety disorders, and eating disorders. Moreover, a dimensional assessment was made with the Beck depression inventory (BDI), a self-reported questionnaire to measure the presence and severity of depressive symptoms [13].

\section{Molecular analyses}

With the patients' written informed consent, $10 \mathrm{cc}$ of peripheral blood were collected, total genomic DNA purified [14], and genotyping for the BDNF Val66Met-BDNF variant was performed essentially as reported [15]. Genotyping was performed blind to clinical and psychiatric data. 
Power calculation and statistical analysis

All data obtained in the three phases of the study (sociodemographic and clinical recordings, psychiatric assessment, and genetic determinations) were merged in a all-comprehensive database by an independent data-manager, which opened the blind and performed the sample size analysis on a preliminary group, to indicate the minimal number of patients needed in the study, and statistical analyses on definitive data.

On the base of initial 36 patients, a sample size analysis was performed to obtain the required number of subjects to fulfil a desired power of $80 \%$ with significance level at $5 \%$ [16]. Because our primary endpoint was to detect a difference in number of analgesics consumed per month by patients between the two groups (see introduction), we divided $G / G$ from non-G/G subjects and calculated the monthly mean of drug consumption: 21 non-G/G subjects consumed meanly 45.57 doses versus 32.93 doses of 15 G/G subjects ( $t=1.887, P=0.07$, Cohen's $d=0.65)$. These data were used to calculate the sample size. Required sample size resulted to be 87 subjects, respectively, 51 for non-G/G and 36 for $\mathrm{G} / \mathrm{G}$ group.

Descriptive statistics to compare BDNF genotypes were performed using parametric or, when appropriate, nonparametric tests. A stepwise multiple linear regression analysis was employed to identify significant predictors of the monthly drug number in the entire sample. In addition, to well-know indexes of null hypothesis significance testing, effect size measures were also reported to recognize the value of the degree of association among variables, particularly between BDNF polymorphism group and monthly drug number $[17,18]$. Effect sizes were calculated with the phi $(\varphi)$ coefficient [or, when appropriate, Cramér's $\mathrm{V}\left(\varphi_{\mathrm{c}}\right)$ ] for $\chi^{2}$ test, with Cohen's $d$ for Student's $t$ test, with partial Eta squared $\left(\eta_{\mathrm{p}}^{2}\right)$ for the analysis of variance (ANOVA), and with Cohen's $f^{2}$ for multiple regression [19]. Statistical significance was set at $5 \%$.

\section{Results}

A hundred and seven $\mathrm{MOH}$ patients (84 women and 23 men; age, mean \pm SD: $46.741 \pm 11.36$ years) were enrolled in the study. Depending on the type of overused drug, patients were divided into four groups: 29 patients (27.10\%) consumed exclusively triptans, 18 (16.82\%) exclusively non-steroidal antinflammatory drugs (NSAIDs); 27 (25.23\%) exclusively combination drugs (combination), and $33(30.84 \%)$ associated different types of drugs (association). None of the patients consumed opioid drugs; all types of combination drugs consumed by our patients resulted to contain caffeine. By genotyping entire
MOH population, we identified seven cases of harboured A/A genotype (6.54\%), 53 were G/A (49.53\%), and 47 were $\mathrm{G} / \mathrm{G}(43.93 \%)$. Samples were in Hardy-Weinberg equilibrium $\left(\chi^{2}=2.460, d f=1, P=0.12\right.$ for the null hypothesis).

A preliminary analysis was performed to investigate differences in monthly drug numbers between A/A $($ mean \pm SD $54.86 \pm 19.05), \mathrm{A} / \mathrm{G}(49.06 \pm 27.19)$ and $\mathrm{G} / \mathrm{G}(33.83 \pm 14.04)$ group. Due to small sample size in A/A group, we used the Kruskal-Wallis $H$ test, the Mann-Whitney $U$ test, and the Monte Carlo method, a bootstrapping technique with a resampling process that produces nonparametric significance estimates. The three groups differed statistically (among them) in number of monthly used drug [mean rank: A/A, 76.21; A/G, 61.43; $\mathrm{G} / \mathrm{G}, 42.31 ; H=13.317, d f=2, P<0.0001$ (99\% confidence interval $<0.0001-0.001)]$. Pairwise comparisons (significance threshold adjustment with Bonferroni's correction, $P=0.0167)$ showed higher values in $\mathrm{A} / \mathrm{A}$ [mean rank: A/A, 43.00; G/G, 25.19; $U=56.000$, $Z=2.794, P_{2 \text {-tailed }}=0.004(99 \%$ CI $\left.0.003-0.006)\right]$ and $\mathrm{A} / \mathrm{G}$ [mean rank: A/G, 58.82; G/G, 41.12; $U=804.500$, $Z=3.046, P_{2 \text {-tailed }}=0.002(99 \%$ CI $\left.0.001-0.003)\right]$ when compared with $\mathrm{G} / \mathrm{G}$ patients, but no difference between $\mathrm{A} / \mathrm{A}$ and $\mathrm{A} / \mathrm{G}$ [mean rank: A/A, 37.21; A/G, 29.61; $U=138.500, Z=1.082, P_{2 \text {-tailed }}=0.29(99 \%$ CI 0.28 $0.30)]$.

Till now obtained results confirmed our decision to consider together carriers of allele $A$ in the non-G/G group: (1) above-mentioned explorative analysis showed that A/A and $\mathrm{A} / \mathrm{G}$ groups were not different in terms of monthly drug consumption, (2) as expected, a little size of A/A subjects was found, and it makes difficult to perform complex, multivariate statistical analysis with an acceptable degree of reproducibility.

As mentioned, to perform further statistical analyses, the population was divided into two groups: G/G genotype (47 subjects, 43.93\%), homozygotes for valine (allele G), and non-G/G genotype (60 subjects, 56.07\%), carriers of metionine 66 (allele A), either in heterozygosity or in homozygosity.

Clinical characteristics and descriptive statistics for the two groups are illustrated in detail in Table 1. Non-G/G showed a higher consumption of monthly drug number (non-G/G vs. G/G, mean $\pm \mathrm{SD}: 49.73 \pm 26.31$ vs. $33.83 \pm 14.04 ; \quad t=4.011, \quad P<0.0001, \quad d=0.76)$ and longer $\mathrm{MOH}$ duration $(6.43 \pm 7.78$ vs. $3.09 \pm 3.66$; $t=2.944, P=0.004, d=0.56)$ when compared to $\mathrm{G} / \mathrm{G}$ patients. There were no significant differences between $\mathrm{G} / \mathrm{G}$ and non-G/G patient groups in terms of other clinical and socio-demographic features. Moreover, no significant associations were found among the two groups and current occurrence of psychiatric disorders. 
Table 1 Table shows descriptive socio-demographic, headache, and psychiatric data for the entire study sample

\begin{tabular}{|c|c|c|c|c|c|}
\hline & $\begin{array}{l}\text { Non-G/G } \\
(n=60 ; 56.07 \%)\end{array}$ & $\begin{array}{l}\mathrm{G} / \mathrm{G} \\
(n=47 ; 43.93 \%)\end{array}$ & Statistics & & \\
\hline \multicolumn{6}{|l|}{ Gender } \\
\hline Women & $47(43.93 \%)$ & $37(34.58 \%)$ & $\chi^{2}=0.002$ & $P=0.96$ & $\varphi=0.005$ \\
\hline Men & $13(12.15 \%)$ & $10(9.35 \%)$ & & & \\
\hline Age (years) & $47.57 \pm 11.47$ & $44.94 \pm 11.15$ & $t=1.192$ & $P=0.24$ & $d=0.23$ \\
\hline Education (years) & $12.34 \pm 4.40$ & $12.59 \pm 4.09$ & $t=-0.262$ & $P=0.79$ & $d=-0.06$ \\
\hline \multicolumn{6}{|l|}{ Employment } \\
\hline Yes & $38(35.51 \%)$ & $32(29.91 \%)$ & $\chi^{2}=0.263$ & $P=0.61$ & $\varphi=-0.050$ \\
\hline No & $22(20.56 \%)$ & $15(14.02 \%)$ & & & \\
\hline Age of primary headache onset (years) & $16.20 \pm 7.42$ & $15.32 \pm 7.14$ & $t=0.620$ & $P=0.54$ & $d=0.12$ \\
\hline Primary headache duration (years) & $32.07 \pm 11.96$ & $30.43 \pm 14.28$ & $t=0.647$ & $P=0.52$ & $d=0.13$ \\
\hline Age of $\mathrm{MOH}$ onset (years) & $41.53 \pm 11.82$ & $42.66 \pm 12.64$ & $t=0.475$ & $P=0.64$ & $d=-0.09$ \\
\hline MOH duration (years) & $6.43 \pm 7.78$ & $3.09 \pm 3.66$ & $t=2.944$ & $P=0.004$ & $d=0.56$ \\
\hline Monthly headache days & $24.82 \pm 6.07$ & $25.89 \pm 5.70$ & $t=-0.935$ & $P=0.35$ & $d=-0.18$ \\
\hline Monthly drug number & $49.73 \pm 26.31$ & $33.83 \pm 14.04$ & $t=4.011$ & $P<0.0001$ & $d=0.76$ \\
\hline Monthly drug days & $23.63 \pm 6.14$ & $22.17 \pm 6.46$ & $t=1.195$ & $P=0.24$ & $d=0.23$ \\
\hline \multicolumn{6}{|l|}{ Drug type } \\
\hline Triptans & $18(16.82 \%)$ & $11(10.28 \%)$ & $\chi^{2}=0.707$ & $P=0.87$ & $\varphi_{\mathrm{c}}=0.081$ \\
\hline NSAIDs & $10(9.35 \%)$ & $8(7.48 \%)$ & & & \\
\hline Association & $17(15.89 \%)$ & $16(14.95 \%)$ & & & \\
\hline Combination & $15(14.02 \%)$ & $12(11.21 \%)$ & & & \\
\hline \multicolumn{6}{|l|}{ Current psychiatric diagnosis } \\
\hline Present & $45(42.06 \%)$ & $34(31.78 \%)$ & $\chi^{2}=0.096$ & $P=0.76$ & $\varphi=0.030$ \\
\hline Absent & $15(14.02 \%)$ & $13(12.15 \%)$ & & & \\
\hline \multicolumn{6}{|l|}{ Current mood diagnosis } \\
\hline Present & $27(25.23 \%)$ & $22(20.56 \%)$ & $\chi^{2}=0.035$ & $P=0.85$ & $\varphi=-0.018$ \\
\hline Absent & $33(30.84 \%)$ & $25(23.36 \%)$ & & & \\
\hline \multicolumn{6}{|l|}{ Current anxiety diagnosis } \\
\hline Present & $32(29.91 \%)$ & $24(22.43 \%)$ & $\chi^{2}=0.054$ & $P=0.82$ & $\varphi=0.023$ \\
\hline Absent & $28(26.17 \%)$ & $23(21.50 \%)$ & & & \\
\hline \multicolumn{6}{|l|}{ Current eating diagnosis } \\
\hline Present & $6(5.61 \%)$ & $2(1.87 \%)$ & $\chi^{2}=1.257$ & $P=0.26^{*}$ & $\varphi=0.108$ \\
\hline Absent & $54(50.47 \%)$ & $45(42.06 \%)$ & & & \\
\hline \multicolumn{6}{|l|}{ Current double psychiatric diagnosis } \\
\hline Present & $18(16.82 \%)$ & $15(14.02 \%)$ & $\chi^{2}=0.045$ & $P=0.83$ & $\varphi=-0.021$ \\
\hline Absent & $42(39.25 \%)$ & $32(29.91 \%)$ & & & \\
\hline BDI (score) & $13.15 \pm 8.53$ & $12.09 \pm 8.73$ & $t=0.634$ & $P=0.53$ & $d=0.12$ \\
\hline
\end{tabular}

Continuous data are expressed as mean $\pm \mathrm{SD}$, whereas categorical data are frequencies and percentages of total subjects. Differences between non-G/G and G/G patients, with bivariate statistics results, are also illustrated. Bold entries represent the statistical significant differences

$\mathrm{MOH}$ medication overuse headache, BDI beck depression inventory, Monthly drug days number of days per month in which patient assumed at least one dose of drug to treat headache, Drug type triptans (exclusively one, or more, types of triptan), NSAIDs (exclusively one, or more, type of non steroidal antinflammatory drugs), association (use of different types of drug in association); combination (drugs of combination, with more active ingredients)

* Fisher's exact test, two-tailed: $P=0.46$

A two-way analysis of covariance (ANCOVA) was employed to investigate the group effect (two betweensubject factors: "genotype", non-G/G vs. G/G; "drug type", triptans vs. NSAIDs vs. association of drugs vs. drugs of combination) on monthly drug number (dependent variable); monthly headache days and monthly drug days were used as covariate in this analysis to reduce the error variance. The ANCOVA model was highly significant $\left(F_{9,97}=7.149\right.$, 
$P<0.0001)$ and explained more than one-third of variance (adjusted $\left.R^{2}=0.343\right)$. The "drug type" effect was not significant $\left(F_{3,97}=0.383, P=0.77, \eta_{\mathrm{p}}^{2}=0.011\right)$ whereas the "genotype" effect was statistically considerable $\left(F_{1,97}=\right.$ $\left.12.487, P=0.0006, \eta_{\mathrm{p}}^{2}=0.114\right)$; the interaction effect of "genotype" $\times$ "drug type" was also significant $\left(F_{3,97}=\right.$ $\left.2.776, P=0.04, \eta_{\mathrm{p}}^{2}=0.079\right)$ (Fig. 1). Among the covariate, monthly drug days was highly significant $\left(F_{1,97}=16.918\right.$, $\left.P<0.0001, \eta_{\mathrm{p}}^{2}=0.149\right)$ but there was no effect of monthly headache days $\left(F_{1,97}=0.043, P=0.84, \eta_{\mathrm{p}}^{2}=0.000\right)$. Pairwise comparisons [Fisher's least square difference (LSD); significance threshold adjustment for multiple comparison test with Bonferroni's correction (0.05/8): $P=0.00625$ ] showed no genotype difference in patients who consumed triptans $(P=0.74)$ and NSAIDs $(P=0.16)$; on the contrary, when compared with $\mathrm{G} / \mathrm{G}$, non-GG patients had a statistically higher consumption of drug both among those that used association of drugs ( $P=0.009$; this value, however, did not reach the adjusted threshold of significance) and among consumers of drugs of combination $(P<0.0001)$.

As showed in Table 2, in the multiple regression analysis, the Val66Met BDNF polymorphism (Beta $=0.33$, $\left.t=3.669, P<0.0001, f^{2}=0.134\right)$ and the BDI score (Beta $=0.19, t=2.090, P<0.04, f^{2}=0.037$ ) emerged as significant, independent predictors of the monthly drug consumption in $\mathrm{MOH}$ patients. No further variables, considered as possible predictors, entered the regression model (see Table 2).

\section{Discussion}

Consistent with our working hypothesis, monthly drug consumption in $\mathrm{MOH}$ patients resulted to be influenced by

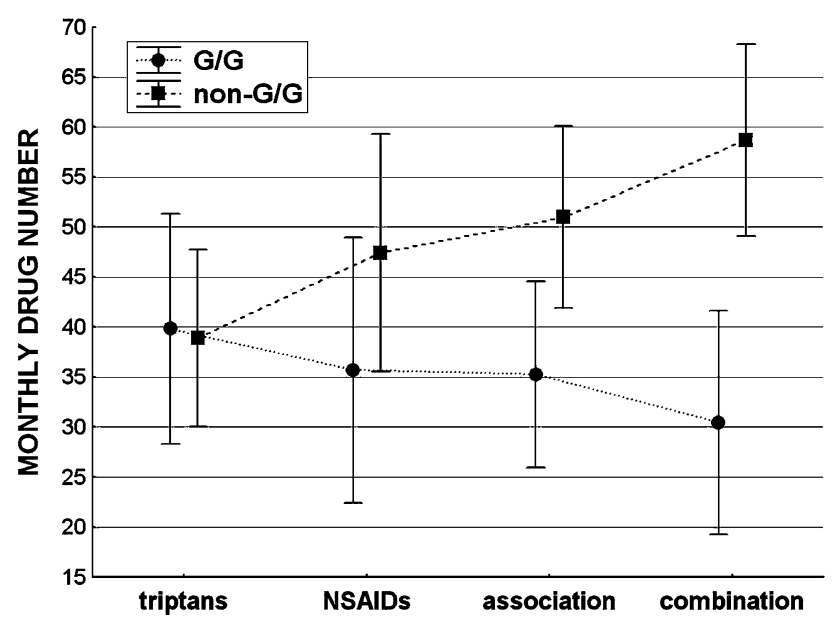

Fig. 1 Interaction effect of "genotype" $\times$ "drug type" on monthly drug number. Markers (black circles and squares) indicate mean, vertical bars denote 0.95 confidence intervals the examined BDNF polymorphism (Fig. 2), both if univariate, and both if multiple regression analyses (to control the association for such possible confounding factors) are performed. The relationship between monthly drug consumption and Val66Met polymorphism was also confirmed by effect size calculation (medium effect at $t$ test, small to medium effect at regression analysis). The fact that a unique BDNF SNP could account for these observed

Table 2 Results of stepwise multiple regression analysis: BDNF polymorphism (G/G genotype) and BDI score (depressive symptoms) are the predictors of monthly drug number in $\mathrm{MOH}$ patients

\begin{tabular}{lrrl}
\hline Variable & Beta & \multicolumn{1}{l}{$t$} & $P$ \\
\hline BDNF gene & $\mathbf{0 . 3 3}$ & $\mathbf{3 . 6 6 9}$ & $<\mathbf{0 . 0 0 0 1}$ \\
BDI & $\mathbf{0 . 1 9}$ & $\mathbf{2 . 0 9 0}$ & $<\mathbf{0 . 0 4}$ \\
MOH duration & 0.12 & 1.242 & 0.22 \\
Drug type & 0.08 & 0.917 & 0.36 \\
Gender & 0.08 & 0.908 & 0.37 \\
Employment & -0.07 & -0.754 & 0.45 \\
Current psychiatric diagnosis & 0.05 & 0.532 & 0.60 \\
Age of headache onset & -0.02 & -0.167 & 0.87 \\
Age & 0.01 & 0.123 & 0.90 \\
\hline
\end{tabular}

Significant predictors are in bold

Stepwise model summary: adjusted $R^{2}=0.137, F_{2,104}=9.424$, $P<0.0001, f^{2}=0.181$

Stepwise model change statistics: first step, variable entered $=$ BDNF gene, $R^{2}$ change $=0.118, \quad F_{1,105}$ to enter/to remove $=14.030$, $P<0.0001, f^{2}=0.134$; second step, variable entered $=\mathrm{BDI}, R^{2}$ change $=0.036, \quad F_{1,104}$ to enter/to remove $=4.368, \quad P<0.04$, $f^{2}=0.037$

$M O H$ medication overuse headache, $B D N F$ brain-derived Neurotrophic factor, $B D I$ beck depression inventory

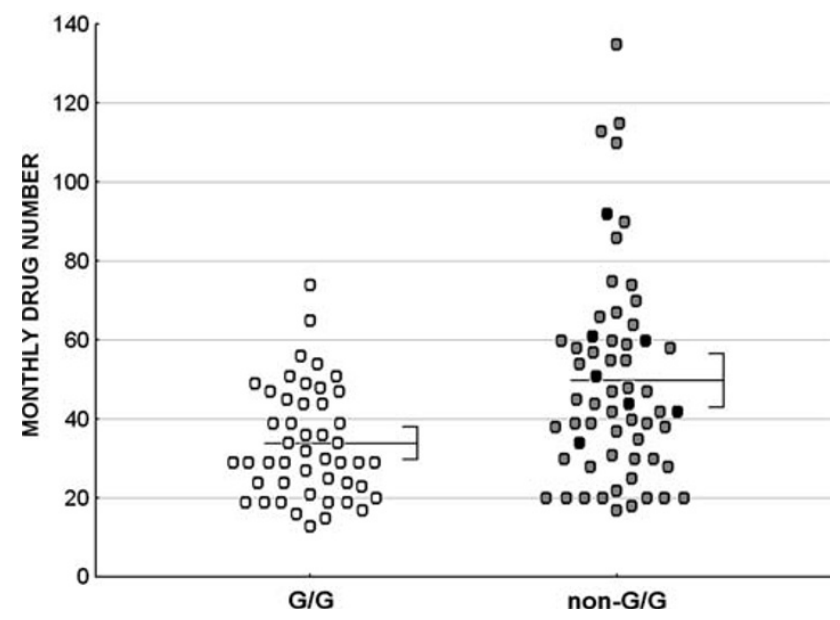

Fig. 2 Monthly drug number in $\mathrm{G} / \mathrm{G}$ and non-G/G patients. Each circle represents an individual patients. In non-G/G group, the black circles show A/A patients, the grey ones with black ring A/G patients. Horizontal bars indicate mean. Vertical bars denote 0.95 confidence intervals 
effects is noteworthy, because drug consumption is a multifactorial variable, surely influenced by many other genetic and environmental factors.

$\mathrm{MOH}$ can be considered as a clinical condition at the boundaries between drug addiction and chronic headache. The hypothetical involvement of BDNF in the pathophysiology of $\mathrm{MOH}$ was suggested by: (1) the association between the Val66Met polymorphism and drug abuse disorders, (2) the role of BDNF in modulating central and peripheral pain. In this study group, non-G/G patients showed a more severe clinical picture, because they had used a higher number of analgesic drugs in the previous 3 months, and had longer disease duration. Showing for the first time a role in $\mathrm{MOH}$, our findings confirm that somehow the examined BDNF polymorphism is involved, as an independent factor, in pathophysiology of this headache condition. Although it might sound too approximate, considering the association between Met66 pro-BDNF and a reduced BDNF activity [7], it is tempting to speculate that in $\mathrm{MOH}$ a lower BDNF activity is a worsening factor. If $\mathrm{MOH}$ is regarded as a drug abuse disorder, our data would agree with previous reports of an association between the Val66Met polymorphism and substance addition [6]. The proneness to abuse (i.e. to have an impulse dyscontrol), previously reported in non-G/G subjects, would be expressed in $\mathrm{MOH}$ patients by higher drug consumption. In light of this hypothesis, the monthly drug consumption could be considered as an important marker of $\mathrm{MOH}$ severity. In fact, although the defining factor in $\mathrm{MOH}$ is the number of days with drug intake, the variability of this value theatrically ranges from 10 to 30; on the contrary, a hypothetic upper limit for variability of monthly drug consumption does not exist and patients could be distributed on a wider range that better reflects their proneness to abuse (and/or headache severity).

Previous studies have attempted to evaluate BDNF role in headache disorders. In particular, it has been demonstrated an increased level of BDNF in cerebrospinal fluid (CSF) of patients with a chronic daily headache (with or without drug overuse), in comparison to healthy controls [20]. Furthermore, it has been shown a reduced level of platelet BDNF in the interictal phase of episodic migraineurs and cluster headache patients, in comparison to healthy controls [21]. More recently, a BDNF elevation in CSF was demonstrated both in chronic migraine and in fibromyalgic patients [22]. These three studies were much different from our research for two reasons: the use of diverse classification criteria in the first study; in general, the different study designs, with the lack of relations among BDNF levels, clinical markers of disease severity, and the patients' BDNF genotype. However, these findings showed the involvement in chronic pain pathophysiology of BDNF up regulation, and might support indirectly our hypothesis that in $\mathrm{MOH}$ patients a BDNF hypo-activity, related to Val66Met polymorphism, does not influence pain severity, but impulsive behaviour.

To the best of our knowledge, Val66Met polymorphism was never tested on pain modulation. Pending specific studies on this topic, only speculations could be made about the chance that the higher drug intake is a consequence of putative BDNF-mediated higher pain sensitivity, or not. However, the pain modulation is linked to BDNF release and up-regulation [23, 24], whereas Val66Met polymorphism is related with a reduction of BDNF activity [7]. These observations, though indirectly, might lend support the idea that BDNF influence on $\mathrm{MOH}$ is played in terms of impulsivity modulation, as in other drug abuse disorder [4], and not pain hypersensitivity.

Moreover, a recent paper analysed influence of Val66Met polymorphism on episodic migraine. Examined polymorphism resulted neither associated to development of migraine (comparing polymorphism frequency in a population of episodic migraineurs with a healthy control group), nor associated with differences in terms of clinical characteristics (within the only migraine group) [25]. Consistent with these data, because Val66Met polymorphism seems to be unable to influence development and clinical features of migraine, we suppose that observed worsen effect of polymorphism on $\mathrm{MOH}$ is likely driven by the A allele related proneness to abuse [6], and, in turn, that the monthly drug consumption in $\mathrm{MOH}$ patients is not necessarily the expression of headache severity.

As reported above, we have also recorded the type of drug consumed by each patient and they were segregated in four groups: triptans, NSAIDs, association of different type of drugs and drugs of combination. It is noteworthy that differences between $\mathrm{G} / \mathrm{G}$ and non-G/G emerged only in the fourth group (drugs of combination, all containing caffeine), and a trend was also found for the association of drugs. If one consider that non-G/G subjects are supposed to have a BDNF hypoactivity [7], and that BDNF is a kind of biological reward for presynaptic neurons [5], our observations could mean that the lack of reward induces non-G/G subjects in the continue search of different type of drugs, or in caffeine seeking (for its psychotropic effect). None of the patients reported to use drugs containing opioids; therefore, we cannot confirm our hypothesis with this class of drugs.

Severity of depressive symptoms (as measured by BDI score) was found as another independent predictor of drug consumption in $\mathrm{MOH}$ patients. The role of depression in headaches has been previously reported and discussed elsewhere [26, 27]. Although a relation between depression and Val66Met polymorphism was frequently found [10], in this study the $\mathrm{G} / \mathrm{G}$ and non-G/G groups neither differ statistically for the severity of depressive symptoms, nor were 
they associated significantly with a psychiatric disorder. Maybe these associations are covered in our population by already described relationship between $\mathrm{MOH}$ and psychiatric comorbidity that could not be further increasable by examined polymorphism. Moreover, observed lack of association suggests that this BDNF polymorphism and depression might have an effect on $\mathrm{MOH}$ monthly drug consumption through different and unrelated mechanisms.

In summary, our results showed an influence of BDNF polymorphism Val66Met on drug consumption in $\mathrm{MOH}$ patients. The polymorphism is likely not related to severer forms of headache, but with a BDNF hypoactivity, in turn related to substance abuse behaviour, consistent with observed higher caffeine seeking. Therefore, aggravating effect of examined polymorphism in $\mathrm{MOH}$ patients could be driven by its proneness to develop an impulsivity dyscontrol, the cornerstone of any substance abuse disorder. In other words, we suppose that $\mathrm{MOH}$ is not only a very severe headache, but also a complicated bio-behavioural disorder, not dissimilar to a kind of addictive disorder, preferentially developed by some migraineurs, aggravated by conditions unrelated to previous headache clinical features; however, related to behavioural impulsivity dyscontrol, as other substance abuse disorders.

Moreover, we would like to highlight that analysis of SNPs within functionally related genes appears as a good tool in headache research to identify mechanisms potentially involved in disease pathogenesis.

Conflict of interest None.

\section{References}

1. Headache Classification Subcommittee of the International Headache Society (2004) The international classification of headache disorders, 2nd edn. Cephalalgia 24(Suppl 1):94-95

2. Lake AE III (2006) Medication overuse headache: biobehavioral issues and solutions. Headache 46:S88-S97

3. Saper JR, Hamel RL, Lake AE III (2005) Medication overuse headache $(\mathrm{MOH})$ is a biobehavioural disorder. Cephalalgia 25:545-546

4. Calabresi P, Cupini LM (2005) Medication-overuse headache: similarities with drug addiction. Trends Pharmacol Sci 26:62-68

5. Binder DK, Scharfman HE (2004) Brain-derived neurotrophic factor. Growth Factors 22:123-131

6. Gratacos M, Gonzalez JR, Mercader JM, de Cid R, Urretavizcaya M, Estivill X (2007) Brain-derived neurotrophic factor Val66Met and psychiatric disorders: meta-analysis of case-control studies confirm association to substance-related disorders, eating disorders, and schizophrenia. Biol Psychiatry 61:911-922

7. Bath KG, Lee FS (2006) Variant BDNF (Val66Met) impact on brain structure and function. Cogn Affect Behav Neurosci 6:79-85

8. Hall D, Dhilla A, Charalambous A, Gogos JA, Karayiorgou M (2003) Sequence variants of the brain-derived neurotrophic factor (BDNF) gene are strongly associated with obsessive-compulsive disorder. Am J Hum Genet 73:370-376
9. Lang UE, Sander T, Lohoff FW, Hellweg R, Bajbouj M, Winterer G, Gallinat J (2007) Association of the met66 allele of brainderived neurotrophic factor (BDNF) with smoking. Psychopharmacology (Berl) 190:433-439

10. Post RM (2007) Role of BDNF in bipolar and unipolar disorder: clinical and theoretical implications. J Psychiatr Res 41:979-990

11. Silberstein SD, Olesen J, Bousser MG et al (2005) International Headache Society, The international classification of headache disorders (ICHD-II)-revision of criteria for 8.2 medicationoveruse headache, 2nd edn. Cephalalgia 25:460-465

12. American Psychiatric Association (2001) Diagnostic and statistical manual of mental disorders, 4th edn. American Psychiatric Press, Washington, DC

13. Beck AT, Steer RA, Brown GK (1996) Manual for the beck depression inventory, 2nd edn. The Psychological Corporation, San Antonio

14. Lahiri DK, Nurnberger JI (1991) A rapid non-enzymatic method for the preparation of HMW DNA from blood for RFLP studies. Nucleic Acids Res 19:5444

15. Neves-Pereira M, Mundo E, Muglia P, King N, Macciardi F, Kennedy JL (2002) The brain-derived neurotrophic factor gene confers susceptibility to bipolar disorder: evidence from a familybased association study. Am J Hum Genet 71:651-655

16. Dupont WD, Plummer WD Jr (1998) Power and sample size calculations for studies involving linear regression. Control Clin Trials 19:589-601

17. Nakagawa S, Cuthill IC (2007) Effect size, confidence interval and statistical significance: a practical guide for biologists. Biol Rev Camb Philos Soc 82:591-605

18. Baghi H, Noorbaloochi S, Moore JB (2007) Statistical and nonstatistical significance: implications for health care researchers. Qual Manag Health Care 16:104-112

19. Cohen J, Cohen P, West SG, Aiken LS (2003) Applied multiple regression/correlation analysis for the behavioral sciences, 3rd edn. Erlbaum, Mahwah

20. Sarchielli P, Alberti A, Gallai B, Coppola F, Baldi A, Floridi A, Gallai V (2002) Brain-derived neurotrophic factor in cerebrospinal fluid of patients with chronic daily headache: relationship with nerve growth factor and glutamate levels. J Headache Pain 3:129-135

21. Blandini F, Rinaldi L, Tassorelli C, Sances G, Motta M, Samuele A, Fancellu R, Nappi G, Leon A (2006) Peripheral levels of BDNF and NGF in primary headaches. Cephalalgia 26:136-142

22. Sarchielli P, Mancini ML, Floridi A, Coppola F, Rossi C, Nardi K, Acciarresi M, Pini LA, Calabresi P (2007) Increased levels of neurotrophins are not specific for chronic migraine: evidence from primary fibromyalgia syndrome. J Pain 8:737-745

23. Pezet S, McMahon SB (2006) Neurotrophins: mediators and modulators of pain. Annu Rev Neurosci 29:507-538

24. Obata K, Noguchi K (2006) BDNF in sensory neurons and chronic pain. Neurosci Res 55:1-10

25. Marziniak M, Herzog A, Mössner R, Sommer C (2008) Investigation of the functional brain-derived neurotrophic factor gene variant Val66MET in migraine. J Neural Transm 115:1321-1325

26. Di Lorenzo C, Sances G, Di Lorenzo G, Rengo C, Ghiotto N, Guaschino E, Perrotta A, Santorelli FM, Grieco GS, Troisi A, Siracusano A, Pierelli F, Nappi G, Casali C (2007) The wolframin His611Arg polymorphism influences medication overuse headache. Neurosci Lett 424:179-184

27. Rossi P, Di Lorenzo G, Malpezzi MG, Di Lorenzo C, Cesarino F, Faroni J, Siracusano A, Troisi A (2005) Depressive symptoms and insecure attachment as predictors of disability in a clinical population of patients with episodic and chronic migraine. Headache 45:561-570 